\title{
Comparação entre os sistemas histomorfológico e de graduação histológica para classificação prognóstica de tumores mamários em cadelas*
}

\author{
Comparison between grading method and histological descriptive \\ system to prognostic classification of mammary tumor in the bitch \\ Tatiane Camacho Mendes ${ }^{1}$, Thomas Normanton Guim ${ }^{1}$, \\ Márcia Feltrin Dias ${ }^{1}$, Josiane Bonel-Raposo ${ }^{2}$ \& Cristina Gevehr Fernandes ${ }^{2}$
}

\begin{abstract}
RESUMO
Este consiste da avaliação comparativa de dois métodos comumente empregados para a classificação de tumores de mama. Compara-se o sistema de classificação histológico-descritivo da Organização Mundial da Saúde (OSM) e o método de graduação histológica proposto por Elston \& Ellis (1991). Carcinomas simples e complexos foram incluídos neste estudo. Todos os carcinomas complexos foram definidos como de grau I $(75 \% ; 24 / 32)$ ou II $(25 \% ; 8 / 32)$. Os carcinomas simples foram graduados em I $(36,4 \% ; 20 / 55)$, II $(32,7 \% ; 18 / 55)$ e III $(30,9 \% ; 17 / 55)$. Os subtipos também foram avaliados e os carcinomas tubulopapilares distribuíram-se nos graus I $(54,1 \% ; 20 / 37)$, II $(37,8 \% ; 14 / 32)$ e III $(8,1 \% ; 3 / 37)$. Os carcinomas sólidos foram graduados em grau II $(33,3 \% ; 3 / 9)$ e III $(66,7 \% ; 6 / 9)$. Nos carcinomas anaplásicos foram encontrados os graus II $(11,1 \% ; 1 / 9)$ e III $(88,9 \%$; 8/9). Conclui-se que o método de graduação de Elston \& Ellis foi mais preciso para determinar o comportamento biológico do que o sistema histológico-descritivo.
\end{abstract}

Descritores: canino, neoplasma, carcinoma mamário.

\section{ABSTRACT}

This paper critically evaluates two methods commonly employed for histological mammary tumor classification. A comparison between the World Health Organization (WHO) histological descriptive system and the Elston and Ellis grading method was done. Mammary complex and simplex carcinomas were included in this study. All tumors considered complex carcinomas were recived grade I $(75 \% ; 24 / 32)$ or grade II $(25 \% ; 8 / 32)$. In simplex carcinomas, grade I $(36,4 \% ; 20 / 55)$, grade II $(32,7 \% ; 18 / 55)$ and grade III $(30,9 \% ; 17 / 55)$ were found. The tumor subtypes were also analyzed and, grade I (54,1\%; 20/37), grade II $(37,8 \% ; 14 / 32)$ and grade III $(8,1 \% ; 3 / 37)$ were observed in tubulopapilar carcinomas. Solid carcinomas show grade II $(33,3 \% / 3 / 9)$ an grade III $(66,7 \% ; 6 / 9)$. Tumors classified as anaplastic carcinomas were graded as II $(11,1 \% ; 1 / 9)$ and III $(88,9 \% ; 8 / 9)$. It was possible to conclude that the Elston and Ellis grading method is mores accurate to determine the biological behavior than the histological descriptive system proposed by Misdorp.

Key words: canine, neoplasm, mammary carcinomas. 


\section{INTRODUÇÃO}

Tumores mamários são vistos freqüentemente em cães, gatos e humanos e são os neoplasmas mais comuns em fêmeas caninas [7,10,1314], sendo diagnosticados tanto tumores malignos como benignos [2,5, 11,14,16]. Podem ser únicos ou múltiplos, e possuem uma histomorfologia intricada, pois ocorrem como tumores epiteliais, mesenquimais e/ou mistos [5].

Devido à sua heterogeneidade, nem sempre a evidência histológica de malignidade, implica em um curso clínico maligno [9-11]. Fatores prognósticos seguros são, então, de grande importância para estimar o risco individual [10].

A proposta do presente estudo foi aplicar dois métodos de classificação histológica para tumores mamários caninos, comparando-os, com o objetivo de evidenciar se ambos se equivalem, se contrapõem ou se complementam, na determinação do potencial maligno dos tumores.

\section{MATERIAIS E MÉTODOS}

Após levantamento prévio da casuística e confecção de novas lâminas histológicas, foram obtidas 87 amostras de tumores mamários caninos, clas- sificados segundo a Organização Mundial da Saúde como carcinomas dos tipos complexo ou simples (subtipos tubulopapilar, sólido e anaplásico) provenientes dos arquivos do Laboratório Regional de Diagnóstico (LRD) da UFPel. Essas mesmas amostras foram graduadas de acordo com o sistema de Elston \& Ellis [3] (Figura 1) o qual quantifica a formação tubular, o pleomorfismo nuclear e o índice mitótico. A graduação histológica é obtida pelo somatório dos escores destes três parâmetros.

\section{RESULTADOS}

Das 87 amostras, 36,8\% (32/87) eram de carcinomas complexos e $63,2 \%$ (55/87) carcinomas simples e, destes, 67,2\% (37/55) carcinomas tubulopapilares, 16,4\% (9/55) sólidos e 16,4\% (9/55) anaplásicos. Verificou-se ainda que, através do sistema de graduação, 50,6\% (44/87) pertenciam ao grau I, 29,9\% (26/ 87) ao grau II e $19,5 \%$ (17/87) ao grau III. Dos 44 tumores grau I, nove possuíam escore 3, doze escore 4 e vinte e três escore 5. Já dos tumores grau II, vinte obtiveram escore 6 e seis escore 7. Dos tumores grau III, doze foram categorizados com escore 8 e cinco escore 9.

\begin{tabular}{cc}
\hline Aspecto & Escore \\
\hline Formação tubular & 1 \\
Boa formação tubular (acima de 75\%) & 2 \\
Moderada formação tubular (entre 10 e 75\%) & 3 \\
Pouco ou nenhuma formação tubular (10\% ou menos) & \\
\hline Pleomorfismo nuclear & 1 \\
Pequeno, células uniformes e regulares & 2 \\
Moderado aumento no tamanho e variabilidade celular & 3 \\
\hline Marcada variação & \\
\hline Contagem mitótica & 1 \\
0 - 7 & 2 \\
8 - 16 & 3 \\
\hline maior que 17 & Grau de malignidade \\
$3-5$ & 1 \\
\hline & III \\
\hline
\end{tabular}

Figura 1. Critérios do método semi-quantitativo para avaliação do grau histológico em carcinomas mamários, proposto por Elston \& Ellis (1991). 
Ao comparar morfologia descritiva e graduação histológica observou-se que, dos 32 tumores complexos, 24 apresentaram grau I de malignidade e oito grau II. Chama-se atenção para o fato de que entre os tumores complexos, não foram encontrados tumores grau III.

Dentre os carcinomas simples (55), 37 eram carcinomas tubulopapilares. Vinte destes foram classificados como grau I, 14 como grau II e 3 como grau III. Já os carcinomas sólidos totalizaram 9 casos, sendo 3 grau II e 6 grau III. Considerando os 9 tumores classificados como carcinomas anaplásicos, um foi classificado como grau II e oito como grau III (Figura 2 e Tabela 1).

\section{DISCUSSÃO}

No presente estudo observou-se, uma distribuição uniforme dos tipos histológicos estudados. Karayannopoulou et al. [6], relataram que de um total de 85 neoplasmas mamários caninos, classificados em carcinoma simples, carcinoma complexo, carci- noma de células fusiformes e carcinoma em tumor benigno, 64,7\% (55/85) eram carcinomas simples e 9,4\% (8/85) eram carcinomas complexos.

Dentre os carcinomas simples, sabe-se que, em cães, os mais comuns são os tubulopapilares [9] e isso foi também observado neste estudo. Os subtipos sólido e anaplásico, neste estudo, expressaram porcentagens iguais de 16,4\% (9/55) cada. Num estudo realizado por Pérez Alenza et al. [12], o subtipo sólido foi o tumor que mais frequientemente apresentou metástases. Já o carcinoma anaplásico, por sua vez, tem um prognóstico desfavorável, pois invariavelmente recidiva e produz metástases [9].

$\mathrm{O}$ escore de um neoplasma é baseado no grau de diferenciação da célula neoplásica e no número de figuras mitóticas do tumor, características estas correlacionadas com a agressividade do tumor. Critérios para o escore dos tumores mamários não são usados sistematicamente em patologia veterinária, o que dificulta muito a predição prognóstica de certos tumores [4]. No presente estudo ao analisar-se os escores, foi ob-

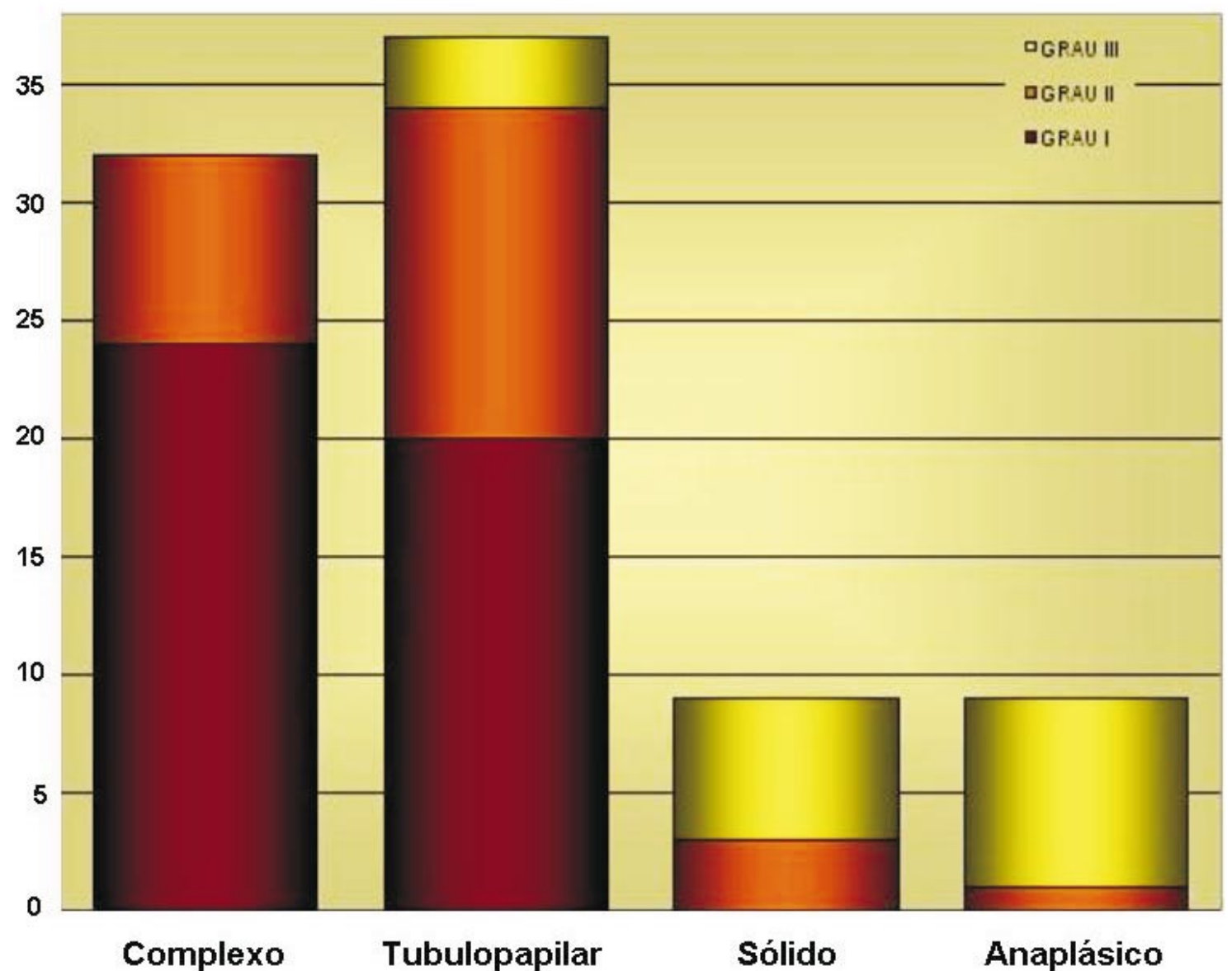

Figura 2. Distribuição dos diferentes tipos de carcinomas mamários caninos e seus respectivos graus de malignidade. 
Tabela 1. Resumo comparativo da distribuição dos carcinomas mamários caninos, de acordo com parâmetros histológicos descritivos e graduação pelos critérios de Elston \& Ellis (1991).

\begin{tabular}{|c|c|c|c|c|c|c|c|c|c|}
\hline \multicolumn{2}{|c|}{ Tipos } & \multicolumn{3}{|c|}{ Grau I } & \multicolumn{2}{|c|}{ Grau II } & \multicolumn{2}{|c|}{ Grau III } & \multirow{2}{*}{ Total (\%) } \\
\hline Escol & & 3 & 4 & 5 & 6 & 7 & 8 & 9 & \\
\hline \multirow[t]{2}{*}{ Carcinoma complexo } & & 5 & 6 & 13 & 6 & 2 & - & - & $32(36,8 \%)$ \\
\hline & Tubulopapilar & 4 & 6 & 10 & 11 & 3 & 3 & & $37(42,4 \%)$ \\
\hline \multirow[t]{2}{*}{ Carcinoma simples } & Sólido & - & - & - & 3 & - & 5 & 1 & $9(10,4 \%)$ \\
\hline & Anaplásico & - & - & - & - & 1 & 4 & 4 & $9(10,4 \%)$ \\
\hline \multicolumn{2}{|c|}{ Total } & 9 & 12 & 23 & 20 & 6 & 12 & 5 & 87 (100\%) \\
\hline
\end{tabular}

servado que a maioria dos tumores, 49,4\% (43/87), encontrava-se concentrada entre os escores 5 e 6 , que são os escores limítrofes entre os graus I e II, e o restante estava distribuído, de forma mais proporcional, entre os outros escores.

Quando se compararam os dois sistemas de classificação, histologia-descritiva e grau de malignidade histológica, observou-se que todos os carcinomas complexos apresentavam-se localizados no grau I $(75 \% ; 24 / 32)$ e II $(25 \% ; 8 / 32)$. Os dados encontrados por Karayannopoulou et al. [6], mostram uma distribuição dos carcinomas complexos semelhante a do presente estudo, no qual $87,5 \%$ (7/8) foram classificados como grau I, $12,5 \%$ (1/8) como grau II e nenhum como grau III. Foi então verificado que, os carcinomas complexos realmente possuem baixo grau de malignidade [9].

Os carcinomas simples distribuíram-se de forma proporcional pelos três graus, com $36,4 \%$ (20/55) no grau I, 32,7\% (18/55) no grau II e 30,9\% (17/55) no grau III, porém, quando os subtipos de carcinomas simples foram submetidos à graduação histológica, evidenciou-se que os tubulopapilares distribuíam-se pelos três graus, principalmente no grau I $(54,1 \%$; $20 / 37)$, seguido pelo grau II $(37,8 \% ; 14 / 32)$ e grau III $(8,1 \% ; 3 / 37)$. Isto reforça o fato de que, dentre os carcinomas simples, o tubulopapilar deve ser o subtipo histológico de menor malignidade [9]. Não havia carcinomas anaplásicos ou sólidos de grau I. Os carcinomas sólidos ficaram distribuídos nos graus II (33,3\%; 3/9) e III $(66,7 \%$; 6/9). Já os carcinomas anaplásicos foram observados nos graus II $(11,1 \%$; $1 / 9)$, e principalmente no grau III $(88,9 \% ; 8 / 9)$. Os resultados obtidos traduzem que estes dois últimos subtipos histológicos de carcinomas simples são realmente os de maior potencial maligno.
Ao levar em conta somente os escores, observou-se que tipos e seus sub-tipos de tumores concentravam-se em escores entendidos como limítrofes nos diferentes graus. Isso foi observado nos carcinomas tubulopapilares. Estes concentraram-se nos escores $5(10 / 37)$ e $6(11 / 37)$ os quais são respectivamente o último escore do grau I e o primeiro do grau II. Já o subtipo sólido revelou escores de 6 a 9, embora o escore 8, o qual é limítrofe ente o grau II e III tenha caracterizado o maior número de casos. Já nos carcinomas anaplásicos, enquadraram-se com maior freqüência nos escores 8 e 9, ambos do grau III, o que caracteriza o reconhecido potencial maligno real deste tumor [9].

Os dados obtidos neste estudo revelaram que, quando se classificam tumores de mama caninos, a distinção entre os tipos complexo e simples é importante, por esses dois tipos possuírem diferenças prognósticas relevantes $[1,8,9,15]$. Isto fica evidente quando estes tumores são submetidos aos sistemas de graduação histológica, pois a grande maioria dos complexos foi caracterizada como grau I, enquanto os carcinomas simples contemplaram os três graus, sendo que o grau III foi representado principalmente pelos carcinomas sólido e anaplásico, os quais efetivamente apresentam maior malignidade. Observa-se também que a sistemática de escores demonstra de forma mais clara, o potencial de malignidade das amostras estudadas. Isso fica claro quando observa-se carcinomas tubulopapilares concentrados nos escores de 5 a 6 , enquanto os anaplásicos distribuem-se especialmente nos escores 8 e 9 .

Karayannopoulou et al. [6], em seu estudo, avaliaram os animais durante um período de 2 anos após a mastectomia inicial. Neste caso, a morte pelo neoplasma ou por eutanásia devido a metástases, foi 
de 45,9\% (39/85). Destes animais, 94,9\% (37/39) eram carcinomas simples, e nenhum era do tipo complexo. Quando avaliada a graduação dos tumores e a taxa de sobrevivência neste estudo, 46,4\% (13/28) dos tumores eram grau II e 86,7\% (26/30) eram grau III. Nenhum paciente de grau I morreu no período do estudo. Isto revela que há um aumento no risco de morte para pacientes com graus II e III, quando comparados com pacientes de grau I.

Sendo assim observa-se a necessidade deste trabalho ser complementado com estudos prospectivos de avaliação da sobrevida dos pacientes, frente ao tipo de tumor e grau que estes apresentam, para assim poder-se realmente avaliar a importância prognóstica destes métodos de classificação histológica para tumores mamários caninos.

\section{CONCLUSÕES}

Com este estudo conclui-se que estes dois sistemas se complementam, sendo importante seu uso concomitante. Porém, a realização isolada da graduação histológica forneceu informações mais fidedignas.

\section{REFERÊNCIAS}

1 Benjamin S.A., Lee,A.C. \& Saunders W.J. 1999. Classification and behavior of canine mammary epithelial neoplasms based on life-span observations in beagles. Veterinary Pathology. 36: 423-436.

2 Cassali G.D. 2000. Estudo morfológico, imuno-histoquímico e citométrico de tumores mamários da cadela - Aspectos comparativos com neoplasias da mama humana. 80f. Belo Horizonte, BH. Tese (Doutorado) - Programa de Pós-graduação da Escola de Veterinária, Universidade Federal de Minas Gerais.

3 Elston C.W. \& Ellis I.O. 1991. Pathological prognostic factors in breast cancer. I. The value of histological grade in breast cancer: experience from a large study with long-term follow-up. Histopathology. 19: 403-410.

4 Ferreira E., Bregunci G.C., Schmitt F.C. \& Cassali G.D. 2003. Protocol for the anatomopathological examination of canine mammary tumors. Arquivo Brasileiro de Medicina Veterinária e Zootecnia. 55: 105-109.

5 Hellmén E., Moller M., Blankenstein M.A.,Andersson L. \& Westermark B. 2000. Expession of different phenotypes in cell lines from canine mammary spindle-cell tumours and osteosarcomas indicating a pluripotent mammary stem cell origin. Breast Cancer Research and Treatment. 61: 197-210.

6 Karayannopoulou M., Kaldrymidou E., Constantinidis T.C. \& Dessiris A. 2005. Histological grading and prognosis in dogs with mammary carcinomas: aplication of a human grading method. Journal of Comparative Pathology. 133: 246-252.

7 Loar A.S. 1992. Tumores do Sistema Genital e Glândulas Mamárias. In: Ettinger S.J. (Ed). Tratado de Medicina Interna Veterinária. 3.ed. São Paulo: Manole Ltda, pp.1900-1906.

8 Misdorp W. \& Hart A.A.M. 1979. Canine mammary cancer I. Prognosis. Journal of Small Animal Practice. 20: 385-394.

9 Misdorp W. 2002. Tumors of the Mammary Gland. In: Meunten D.J. (Ed). Tumors in Domestic Animals. 4th edn. Iowa: Iowa State Press, pp.575-606.

10 Mulas J.M. de Las, Millán Y. \& Dios R. 2005. A prospective analisis of immunohistochemically determined estrogen receptor $\alpha$ and progesterone receptor expression and host and tumor factor as predictors of disease-free period in mammary tumors of the dog. Veterinary Pathology. 42: 200-212.

11 Okamura Y., Haraguchi T., Morimoto M., Okura M., Une S., Nakaichi M. \& Taura Y. 2004. Expression of a tumorassociataed antigen, RCAS1, in canine mammary tumors. Journal of Veterinary Medical Science. 66: 651-658.

12 Pérez Alenza M.D., Peña L., Nieto A. I. \& Casteño M. 1997. Clinical and pathological prognostic factors in canine mammary tumours. Annali dell'Istituto Superiore di Sanitá. 33: 581-585.

13 Pires M.A., Travassos F.S. \& Pires I. 2003. Neoplasias em canídeos - Um estudo descritivo de 6 anos. Revista Portuguesa de Ciências Veterinárias. 98: 111-118.

14 Simon D., Knebel J.W., Baumgärtner W., Aufderheide M. \& Meyer-Lindenberg A. \& Nolte I. 2001. In vitro efficacy of chemotherapeutics as determined by $50 \%$ inhibitory concentrations in cell cultures of mammary gland tumors obtained from dogs. American Journal of Veterinary Research. 62: 1825-1830.

15 Yamagami T., Kobavashi T., Takahashi K. \& Sugivama M. 1996b. Prognosis for malignant mammary tumors based on TNM and histologic classification. Journal of Veterinary Medical Science. 58: 1079-1083.

16 Yazawa M., Okuda M.,Setoguchi A., Iwabuchi S., Nishimura R.,Sasaki N., Masuda K.,Ohno K. \& Tsujimoto

H. 2001. Telomere length and telomerase activity in canine mammary gland tumors. American Journal of Veterinary Research. 62: 1539-1543.

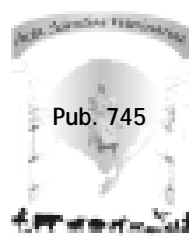

